\title{
A systematic review of interventions conducted in clinical or community settings to improve dual- task postural control in older adults
}

This article was published in the following Dove Press journal:

Clinical Interventions in Aging

25 March 2014

Number of times this article has been viewed

\author{
Maayan Agmon' \\ Basia Belza ${ }^{2}$ \\ Huong Q Nguyen ${ }^{2,3}$ \\ Rebecca G Logsdon ${ }^{2}$ \\ Valerie E Kelly ${ }^{4}$ \\ 'The Cheryl Spencer Department \\ of Nursing, Faculty of Social Welfare \\ and Health Studies, University of \\ Haifa, Israel; ${ }^{2}$ School of Nursing, \\ University of Washington, Seattle, WA, \\ USA; ${ }^{3}$ Department of Research and \\ Evaluation, Kaiser Permanente, CA, \\ USA; ${ }^{4}$ School of Medicine, University \\ of Washington, Seattle, WA, USA
}

Correspondence: Maayan Agmon University of Haifa, Abba Hushi I, Haifa, Israel

Tel +972 54900 I609

Fax+97248288017

Email agmon.mn@gmail.com
Background: Injury due to falls is a major problem among older adults. Decrements in dualtask postural control performance (simultaneously performing two tasks, at least one of which requires postural control) have been associated with an increased risk of falling. Evidence-based interventions that can be used in clinical or community settings to improve dual-task postural control may help to reduce this risk.

Purpose: The aims of this systematic review are: 1) to identify clinical or community-based interventions that improved dual-task postural control among older adults; and 2) to identify the key elements of those interventions.

Data sources: Studies were obtained from a search conducted through October 2013 of the following electronic databases: PubMed, CINAHL, PsycINFO, and Web of Science.

Study selection: Randomized and nonrandomized controlled studies examining the effects of interventions aimed at improving dual-task postural control among community-dwelling older adults were selected.

Data extraction: All studies were evaluated based on methodological quality. Intervention characteristics including study purpose, study design, and sample size were identified, and effects of dual-task interventions on various postural control and cognitive outcomes were noted.

Data synthesis: Twenty-two studies fulfilled the selection criteria and were summarized in this review to identify characteristics of successful interventions.

Limitations: The ability to synthesize data was limited by the heterogeneity in participant characteristics, study designs, and outcome measures.

Conclusion: Dual-task postural control can be modified by specific training. There was little evidence that single-task training transferred to dual-task postural control performance. Further investigation of dual-task training using standardized outcome measurements is needed.

Keywords: physical therapy, balance, walking, motor learning, fall prevention

\section{Introduction}

In 2020, one out of five people in western countries will be 70 years of age or older. ${ }^{1}$ Healthy aging is accompanied by changes in sensory and cognitive domains that may lead to balance and gait impairments. ${ }^{2,3}$ Balance and gait impairments, in turn, contribute to recurrent falls, which are related to increased mortality and morbidity. ${ }^{4}$ Thirty percent of adults over age 65 , and $50 \%$ of those over age 85 , are likely to have at least one fall. ${ }^{5,6}$ Consequently, finding effective ways to decrease falls in the elderly may reduce disability and increase life expectancy. ${ }^{7}$

Although falls are multifactorial, ${ }^{8}$ impaired postural control is one important factor contributing to falls. Postural control is defined as the ability to control the body's position in space for the purposes of stability and orientation, ${ }^{9}$ and is critical 
during standing balance and walking tasks. Much research has focused on the interplay between postural control and cognition, ${ }^{10}$ using dual-task postural control paradigms to examine this relationship. ${ }^{11}$ Dual-task performance refers to the ability to conduct two tasks simultaneously, with dual-task postural control referring to situations when at least one of the tasks involves postural control, such as walking while talking on the phone or while holding a tray. Evaluating dual-task performance is a complex process as it involves the evaluation of each task performed independently as well as in combination. One way of analyzing the performance is by calculating the dual-task cost, defined as the decline in dualtask compared to single-task performance of a task. ${ }^{12-14}$

Changes associated with aging may lead to deterioration with the performance of each individual task as well as with the dual-task combination. For example, the gait pattern is affected by age, with reduced stride length and gait speed as well as increased lateral sway and stride to stride variability among older adults. ${ }^{15}$ Executive function is a set of cognitive skills required in order to plan, monitor, and conduct goal-directed complex actions, ${ }^{16}$ and an important aspect of executive function that tends to deteriorate with aging is the ability to divide or switch attention between the different tasks. ${ }^{17,18}$ This ability is critical for dual-task performance. In older adults, dual-task postural control deficits have been associated with declines in cognitive function ${ }^{19,20}$ and increased risk for falls. ${ }^{21-23}$

Recent studies have demonstrated the potential for modification of dual-task performance among the elderly. ${ }^{24,25}$ Specifically, the ability to divide attention between two tasks in order to conduct them simultaneously is modifiable following training. ${ }^{26,27}$ An important aspect of effective motor learning is training specificity, which refers to training a specific task through repetitive exercises in order to achieve improvements in that task. ${ }^{28}$ Training in dual-task performance is more complicated than training a discrete movement under single-task condition, ${ }^{29}$ and the level of specificity required to improve dual-task performance is still unknown. Moreover, dual-task postural control performance is influenced by the types of tasks, their difficulty, and the outcome measured. ${ }^{30,31}$

There are a growing number of interventions aimed at improving dual-task postural control in healthy older adults. Wollesen and Voelcker-Rehage ${ }^{24}$ performed a systemic review of the dual-task literature to examine the effects of specific versus general training and task combination on dualtask performance. They concluded that dual-task training is more effective than single-task for improving dual-task standing performance, whereas both dual-task and singletask training improved dual-task walking. The current review extends this effort by examining how the application of motor learning principles, such as training specificity, setting, dose, duration, and intensity, may impact the efficacy of dual-task interventions. A better understanding of the effective elements of previous training trials can inform future dual-task interventions designed to improve mobility and reduce fall risk in older adults. Thus, the aims of this systematic review are to: 1) examine the effectiveness of different interventions on dual-task postural control among healthy older adults; and 2) identify key elements of training protocols that effectively improve dual-task postural control in older adults.

\section{Methods}

\section{Data sources and searches}

A systematic search was performed of the following computerized electronic databases: PubMed (January 1966 through October 2013), CINAHL (January 1982 through October 2013), PsycINFO (January 1900 through October 2013), PEDro (January 1929 through October 2013), and Web of Science (January 1900 through October 2013). Search terms included combinations of the following key words: "dual-task"; "older adults" or "elderly"; "treatment" or "intervention" or "therapy" or "rehabilitation"; "gait" or "balance" or "postural control". References found by a manual search in identified articles were also reviewed and included as appropriate.

\section{Inclusion criteria and study selection}

To be included in this systematic review, a study had to meet the following criteria: 1) participants defined as healthy adults aged 60 years or older; 2 ) interventions were conducted in a community or clinical setting; 3 ) interventions required a minimum of 180 minutes of training over at least 3 total days; 4) dual-task postural control was measured as an outcome; and 5) the publication was written in English. Exclusion criteria included participants with a specific neurologic disorder, such as Parkinson's disease, Alzheimer's disease, dementia, or stroke.

Two reviewers (MA, VEK) screened the abstract search results and decided independently, based on inclusion and exclusion criteria, which studies to include. Results were compared and, when reviewer decisions differed, the full article was reviewed and evaluated to obtain agreement.

\section{Data extraction and quality assessment}

Reviewers, who were not blinded to the author or the journal, assessed the quality of each included study in terms 
of the grade of recommendation and the level of evidence provided using the scoring protocol developed by Portney and Watkins. ${ }^{32}$ This scale includes ten levels of evidence divided into four levels of recommendations. The highest level of evidence is a meta-analysis and the lowest level is expert opinion.

Studies were summarized according to the following characteristics: methodological quality and level of evidence; study design; sample size; sample characteristics (age and sex); key characteristics of the training protocols (training specificity, content, setting, intensity); assessment time points; outcome measures (for postural control task and concurrent cognitive or motor task); and results. Data synthesis using a meta-analysis was not possible because of the variety of study designs, methodologies, and outcomes measured.

\section{Results}

The literature search yielded 162 publications that were screened, with 26 publications reviewed in full. Twenty-two publications met the inclusion criteria and were included in this review (Figure 1). Publications were excluded based on the following criteria: 1) not intervention trials (93 publications); 2) population was not appropriate (eg, included younger adults or older adults with a neurologic disorder; 43 publications); 3) did not measure dual-task performance as an outcome (3 publications); or 4) not written in English (1 publication).

\section{Methodological quality and level of evidence}

Twenty-two publications were included in this review, with two publications from the same intervention trial (Table 1).

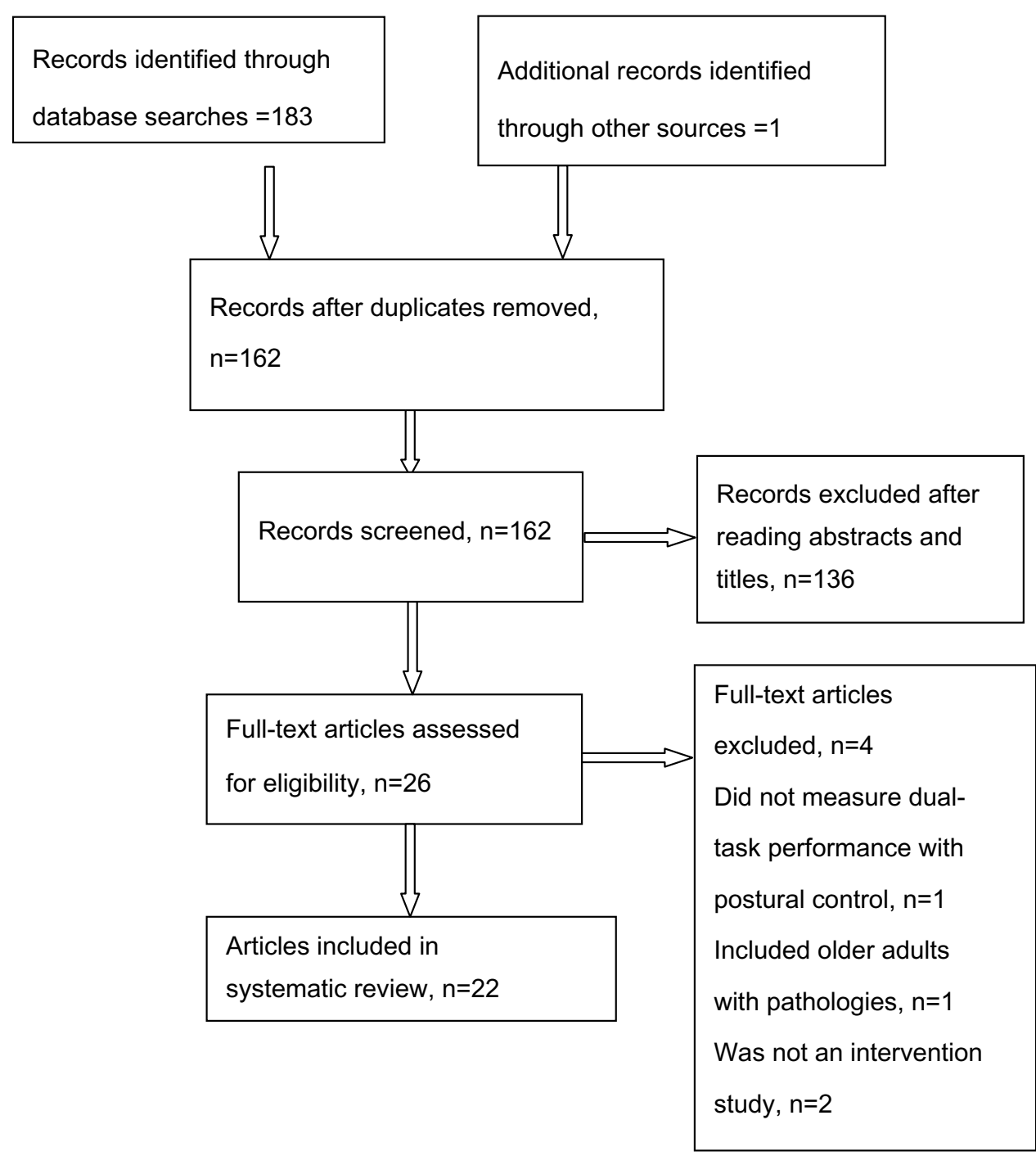

Figure I Flowchart of systematic literature search. 
Table I Level of evidence $(n=22)$

\begin{tabular}{|c|c|c|}
\hline Study & $\begin{array}{l}\text { Level of } \\
\text { evidence }^{32}\end{array}$ & Design \\
\hline Hiyamizu et al, $2012^{33}$ & $\mathrm{Ib}$ & $\mathrm{RCT}$ \\
\hline Li et al, $2010^{34}$ & $\mathrm{Ib}$ & $\mathrm{RCT}$ \\
\hline Melzer et al, $2013^{35}$ & $\mathrm{Ib}$ & $\mathrm{RCT}$ \\
\hline Mozolic et al, 201 I 36 & $\mathrm{Ib}$ & $\mathrm{RCT}$ \\
\hline Silsupadol et al, 2009A ${ }^{26}$ & $\mathrm{Ib}$ & $\mathrm{RCT}$ \\
\hline Silsupadol et al, 2009B ${ }^{27}$ & $\mathrm{Ib}$ & $\mathrm{RCT}$ \\
\hline Trombetti et al, 201 I 37 & Ib & $\mathrm{RCT}$ \\
\hline Yamada et al, 20I I 38 & $\mathrm{Ib}$ & $\mathrm{RCT}$ \\
\hline Donath et al, $2013^{39}$ & $2 b$ & Preliminary RCT \\
\hline Granacher et al, $2010^{40}$ & $2 b$ & Preliminary RCT \\
\hline Hall et al, 2009' & $2 b$ & Preliminary RCT \\
\hline Pichierri et al, $2012^{42}$ & $2 b$ & Preliminary $\mathrm{RCT}$ \\
\hline Plummer-D'Amato et al, $2012^{43}$ & $2 b$ & Preliminary $\mathrm{RCT}$ \\
\hline Uemura et al, $2012^{44}$ & $2 b$ & Preliminary RCT \\
\hline Verghese et al, $2010^{45}$ & $2 b$ & Preliminary RCT \\
\hline You et al, $2009^{46}$ & $2 b$ & Preliminary $\mathrm{RCT}$ \\
\hline Agmon et al, $2012^{47}$ & 3 & $\begin{array}{l}\text { Uncontrolled pretest } \\
\text { to posttest }\end{array}$ \\
\hline Bisson et al, $2007^{48}$ & 3 & $\begin{array}{l}\text { Controlled pretest } \\
\text { to posttest }\end{array}$ \\
\hline Lajoie et al, $2004^{49}$ & 3 & $\begin{array}{l}\text { Controlled pretest } \\
\text { to posttest }\end{array}$ \\
\hline Melzer et al, $2009^{50}$ & 3 & Case control \\
\hline Toulotte et al, $2006^{51}$ & 3 & $\begin{array}{l}\text { Uncontrolled pretest } \\
\text { to posttest }\end{array}$ \\
\hline Silsupadol et al, $2006^{52}$ & 4 & Case series \\
\hline
\end{tabular}

Notes: Levels of evidence: Ia, Systematic review of RCTs; Ib, Individual RCT with narrow confidence interval; 2a, Systematic review of cohort studies; $2 b$, Individual cohort study or low quality RCT; 3, Individual case-control or pretest-posttest study; 4, Case series or poor quality cohort and case-control studies; 5, Expert opinion.

Abbreviation: RCT, randomized clinical trial.

In terms of level of evidence, eight were considered to be level $1 \mathrm{~b}$ (randomized clinical trials with narrow confidence intervals) and eight were considered to be $2 \mathrm{~b}$ (low quality randomized clinical trials). These sixteen studies $^{26,27,33-46}$ had some methodological weaknesses; only one study included an intention-to-treat analysis, ${ }^{37}$ and only four (two from the same trial) incorporated blinded assessors. ${ }^{26,27,43,45}$ The other six were classified as level 3 (case-control or cohort) ${ }^{47-51}$ and level 4 (case series). ${ }^{52}$

\section{Sample characteristics}

Studies were included in this review only if the participants were adults aged 60 years or older. Sample size ranged from three (one per group) ${ }^{52}$ to $134 .{ }^{37}$ The participants were predominantly female.

Sample characteristics varied across studies. Physical and cognitive functioning and the tests used to assess these characteristics varied. Most studies evaluated cognitive status using the mini-mental state examination with inclusion criteria typically based on scores of 24 out of 30 points. ${ }^{26,27,41,46,48,50-52}$ Physical status was defined using performance-based tests, such as the Berg Balance Scale, ${ }^{26,27,35}$ Tinetti Test, ${ }^{37}$ Mini-Balance Evaluation Systems Test (MiniBEST) ${ }^{47}$ or the Dynamic Gait Index, ${ }^{41}$ as well as self-reported walking abilities. ${ }^{26,27,35,38,40,43-45,47-52}$ Fall history was specified only in four studies. ${ }^{41,46,51,52}$ Living situation also varied across studies, though most studies involved participants who lived independently in the community (Table 2).

\section{Training parameters of interventions}

The training protocols incorporated in these studies varied with respect to training specificity (Table 3). Both single-task and dualtask approaches were used, and dual-task training incorporated various task combinations. Of the studies incorporating dualtask interventions, ${ }^{26,27,33-38,43-46,52}$ eleven included postural control tasks as one of the tasks, ${ }^{26,27,33,35-38,43,44,46,52}$ while two trained dualtask performance on two cognitive tasks. ${ }^{34,45}$ The other nine studies ${ }^{39-42,47-51}$ assessed the effects of single-task postural control training on dual-task postural control.

The studies that trained dual-task postural control used different combinations of tasks, with different levels of difficulty in both the cognitive and the postural tasks. For example, Trombetti et $\mathrm{al}^{37}$ combined walking as the postural control task with a variety of motor (eg, handling musical instruments) and cognitive (eg, responding to changes in the beat of the music) tasks. Plummer-D'Amato et $\mathrm{al}^{43}$ trained three different postural control domains (balance, gait, and agility) in combination with four different cognitive tasks (random number generation, word association, backward recitation of words or number sequences, and working memory).

Because dual-task performance can be modified by focusing attention on one task or another, the effect of dual-task training may be influenced by the instructions provided. ${ }^{53}$ In this review, only the three studies conducted by Silsupadol et al ${ }^{26,27,52}$ specifically examined the impact of different instructions on dual-task postural control. These studies compared the effect of two sets of instructions variable priority instructions and fixed priority instructions on dual-task performance following the same training. Variable priority instructions required the participant to focus on one task at a time (either the postural or the cognitive task) while the fixed priority instructions required the participant to focus on both the postural and cognitive tasks at all times.

Interventions were conducted either one-onone $26,27,36,45,46,48,49,52$ or in group settings (four to 24 people per group), ${ }^{33-35,37-44,47,50,51}$ with varying levels of supervision. 


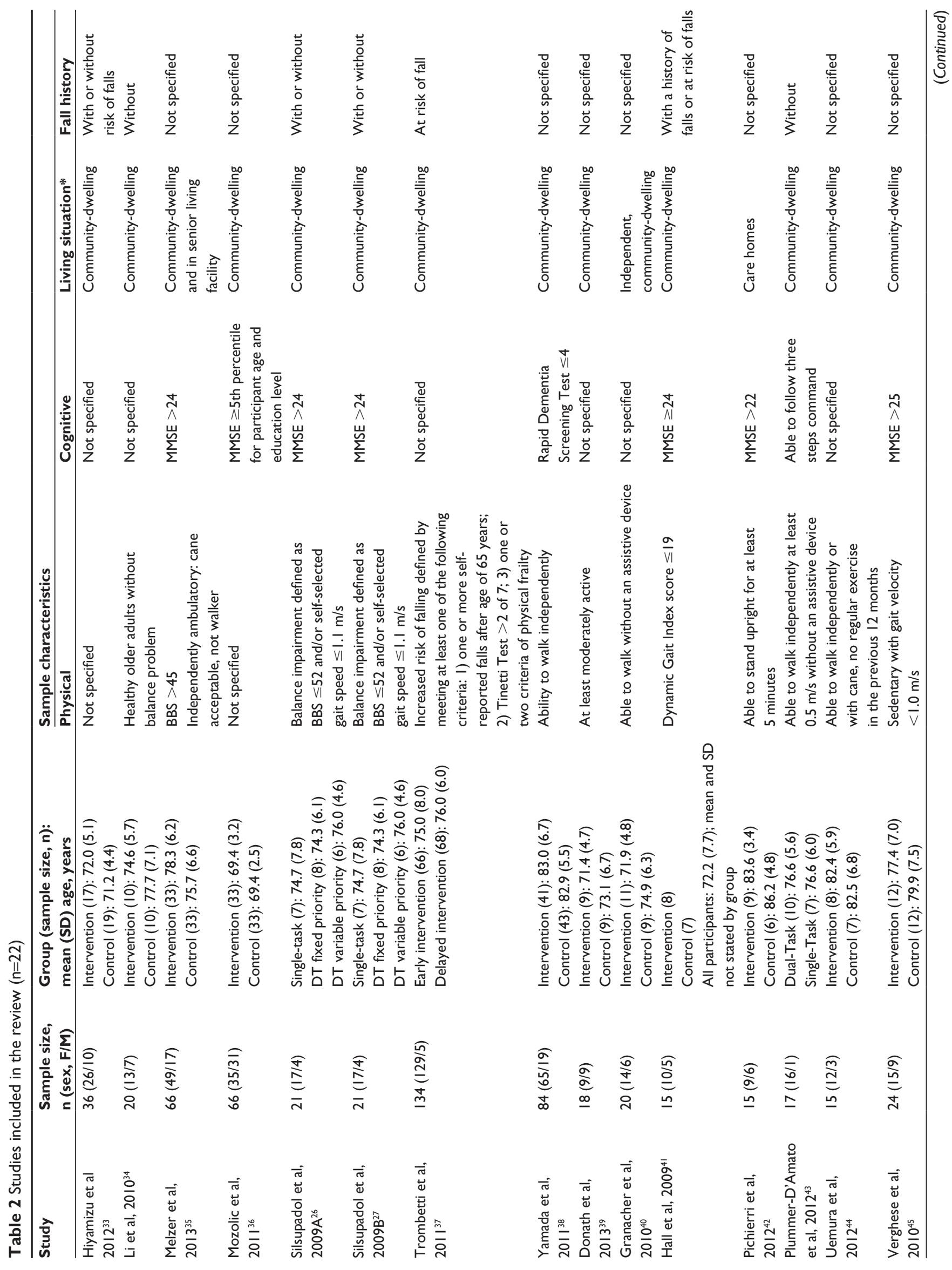




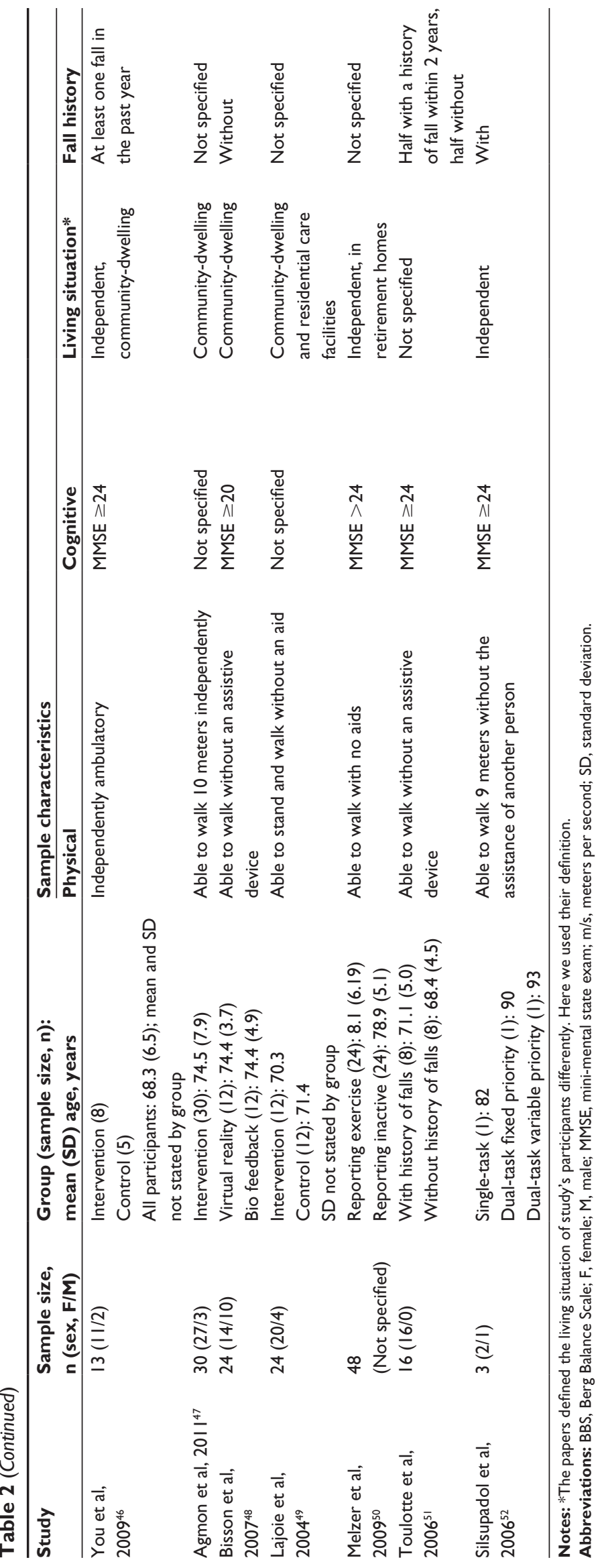

There is no indication that one approach was more effective than the other.

\section{Outcome measures}

Balance and walking under both single-task and dualtask conditions was assessed using a variety of measures (Table 4). Therefore, there was no common set of standardized measures that could be used to compare changes in dual-task postural control across the studies (Table 2). Measures of single-task balance and walking included the Berg Balance Scale, the Dynamic Gait Index, the Timed Up and Go test, postural sway, and various gait parameters (speed, gait stability, center of mass or center of pressure, and variability) assessed during both simple and complex walking tasks. In addition, a number of studies incorporated measures of balance confidence or self-efficacy (Activities-specific Balance Confidence Scale, Falls Efficacy Scale) or function (Late Life Function and Disability Index) to examine the effects of training protocols on balance self-efficacy and functioning in daily life.

Measures of dual-task balance and walking included postural sway and gait parameters (speed, stability, variability). For dual-task balance and walking, performance on a variety of concurrent cognitive and motor tasks was also assessed. Examples of motor tasks included separating two linked rings or throwing and catching a ball. Examples of cognitive tasks included arithmetic tasks (eg, serial-3 subtractions), working memory tasks (eg, n-back test), or choice reaction time tasks (eg, auditory Stroop test). Dualtask performance was also evaluated using the dual-task cost calculation. In the studies that trained dual-task postural control, some assessed the efficacy of the intervention using trained task combinations ${ }^{40}$ while others measured at least one novel task. ${ }^{26,27,52}$

\section{Dual-task performance changes}

From the 22 publications included in this review, 18 demonstrated improvement in some aspect of dual-task performance ${ }^{26,27,33-40,42,44-49,51,52}$ whereas four did not. ${ }^{41,43,50,54}$ Of those showing improvement, three studies ${ }^{26,27,52}$ demonstrated improvement for both the postural control task and the concurrent cognitive or motor task. Seven studies demonstrated improvements in the dual-task cost for either postural control tasks or cognitive tasks, but not both. ${ }^{37,40,46-49,51}$ The other eight did not measure dual-task cost and demonstrated improvement on only one aspect of the tasks combination. ${ }^{33-36,38,39,42,44}$ 


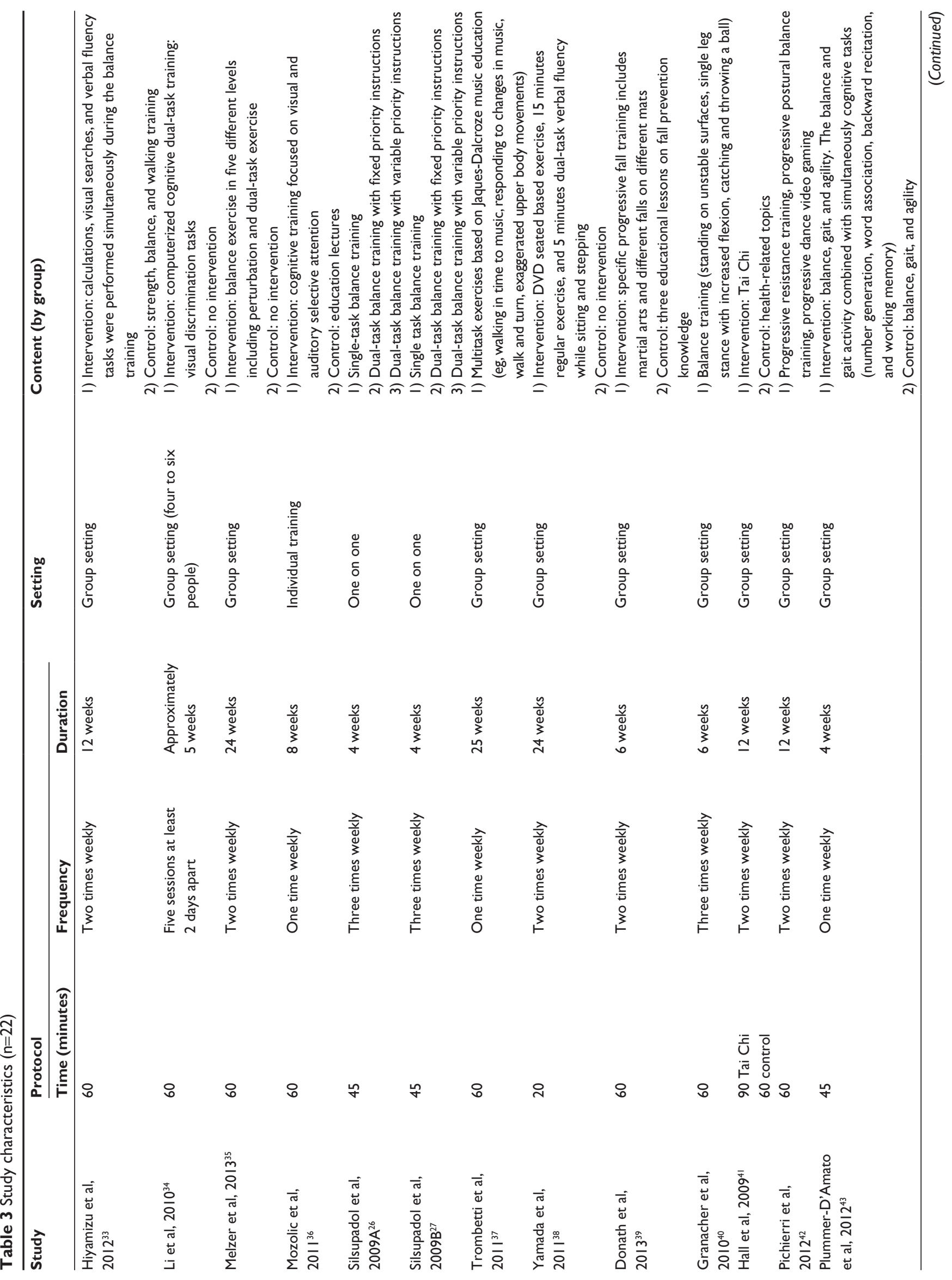




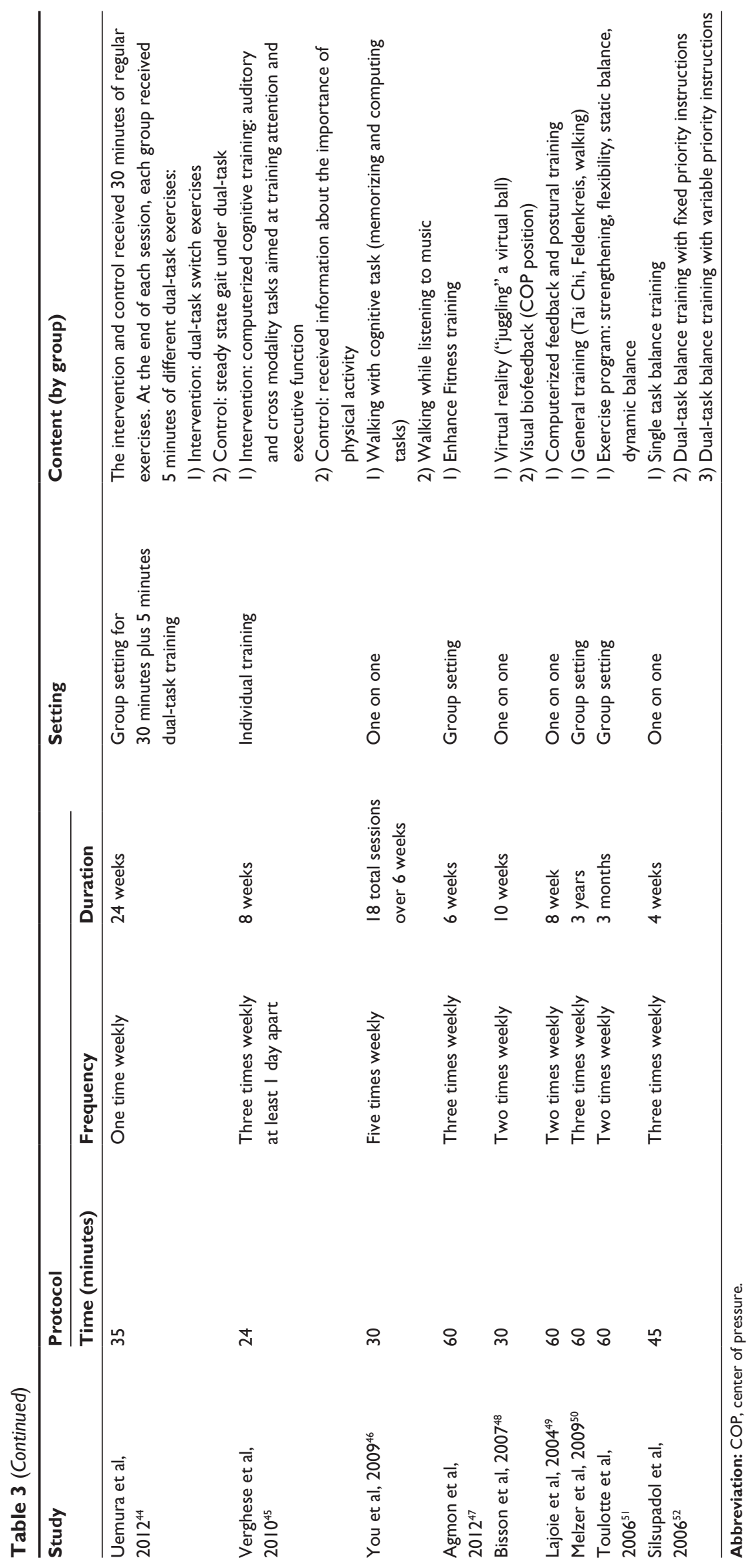




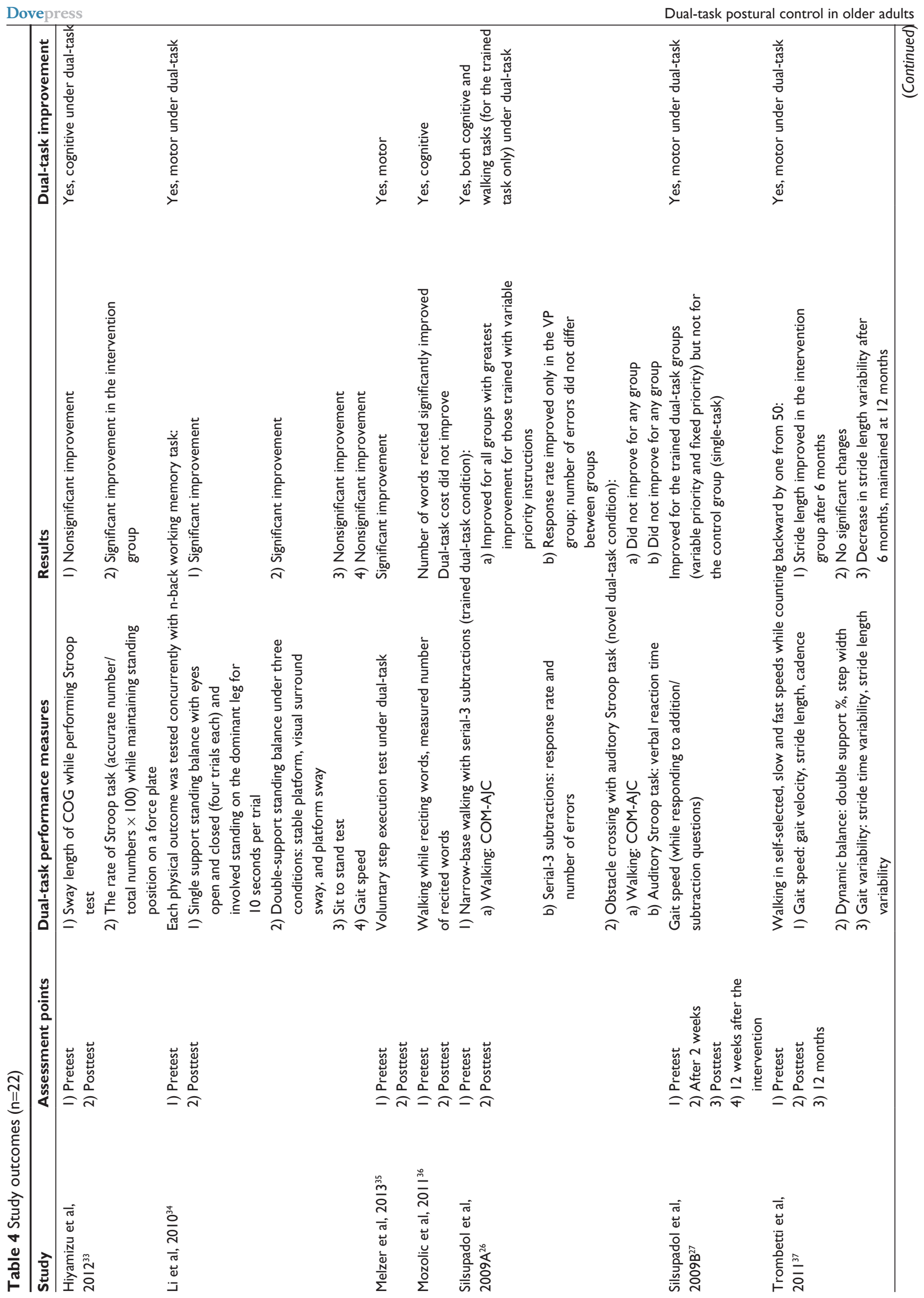



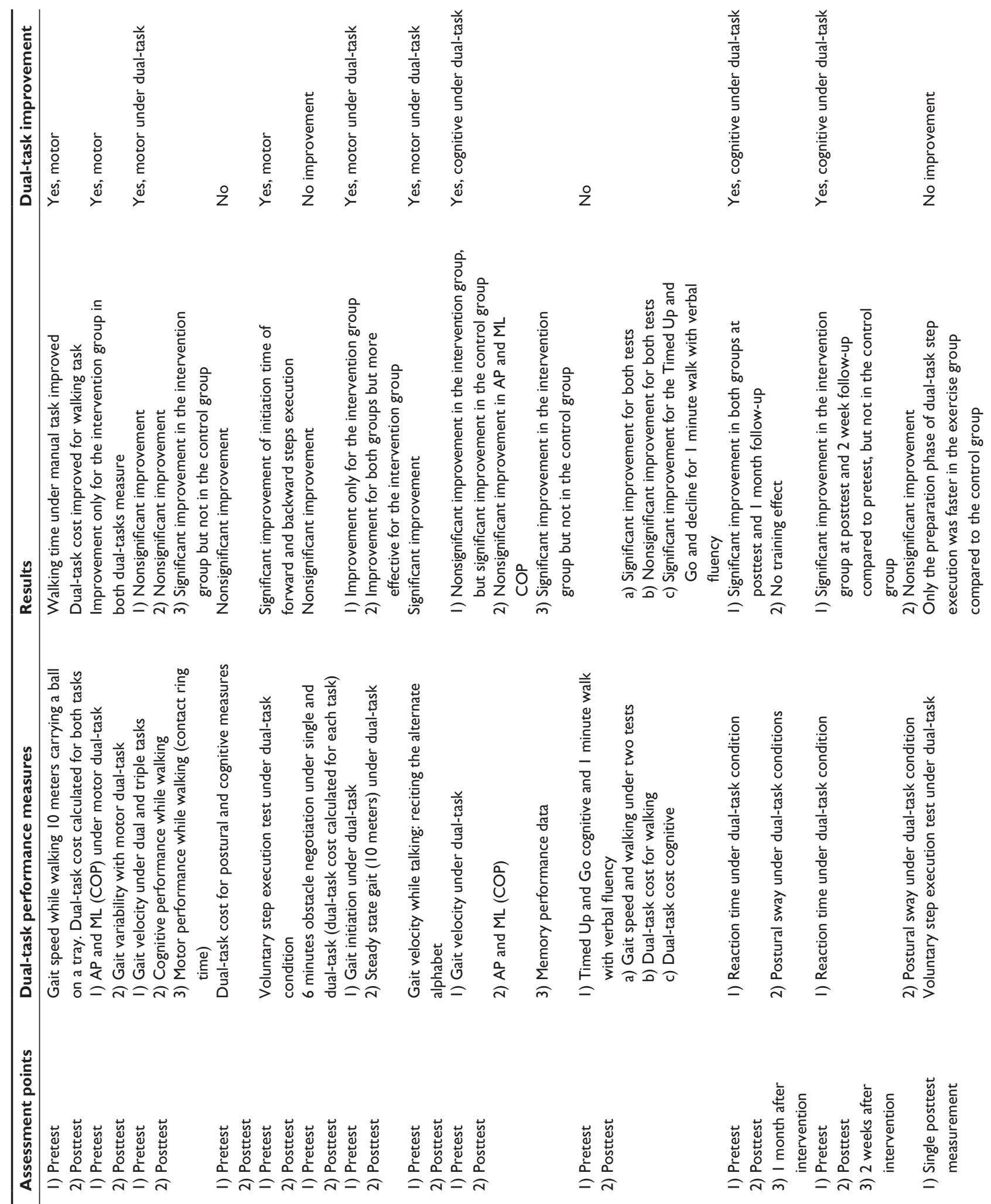

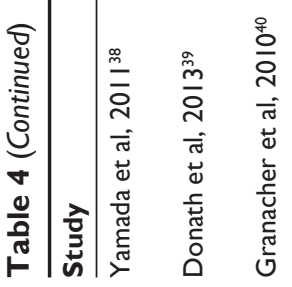
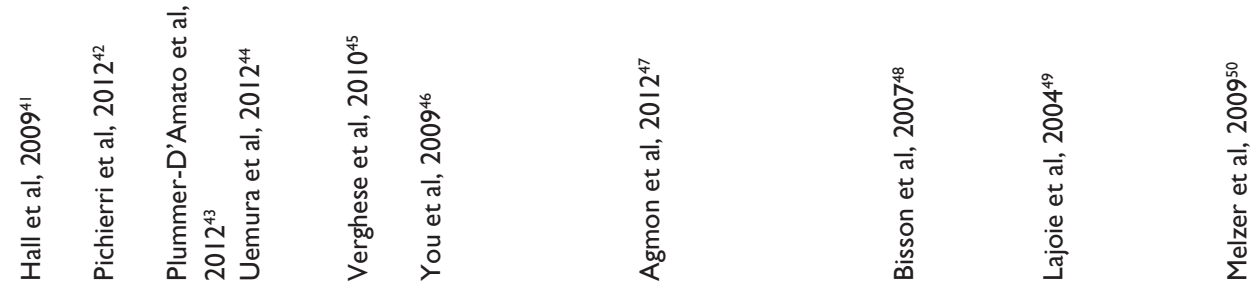

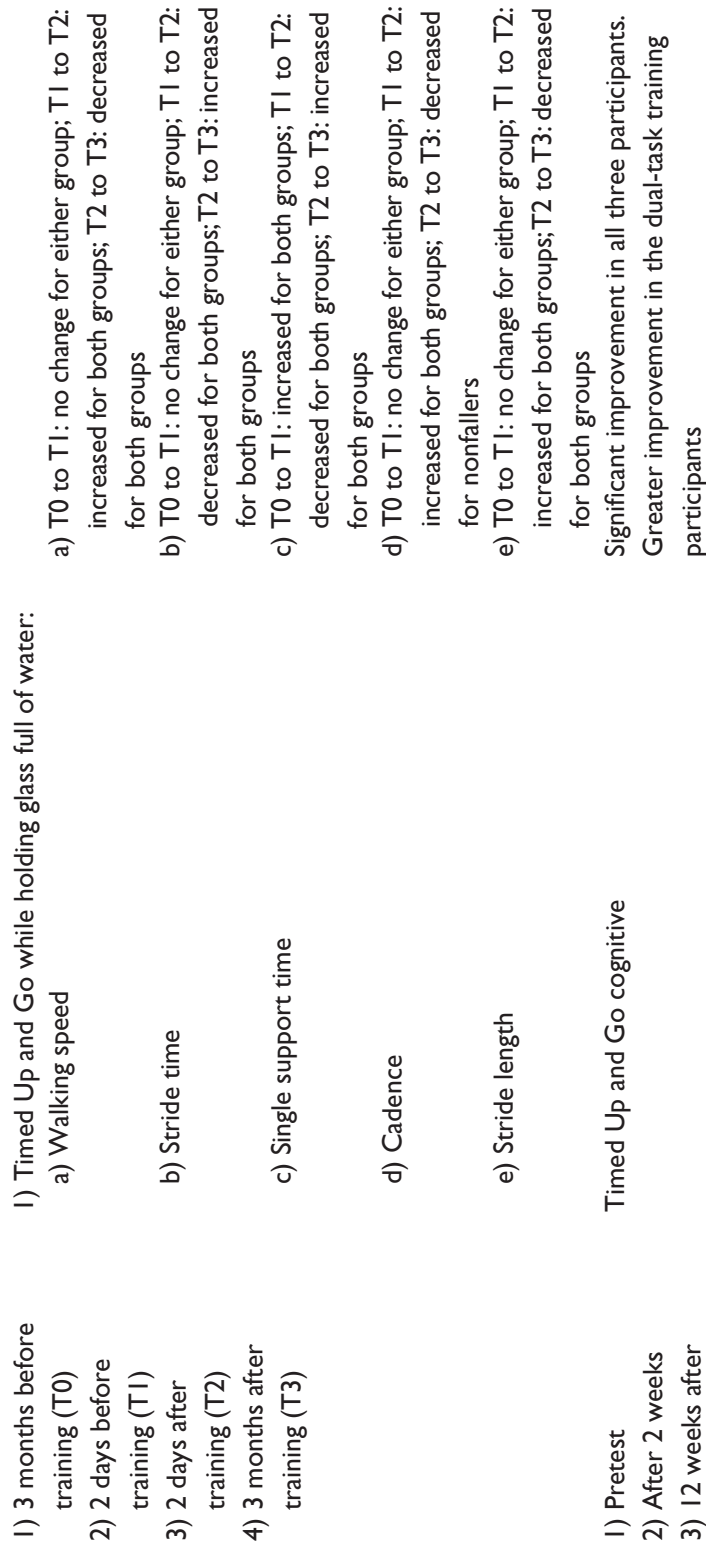

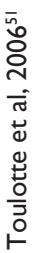

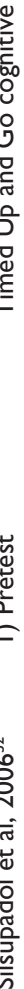

\section{Retention}

Most studies measured outcomes immediately before and after the intervention, with only five studies examining retention of improvements at different time points after the end of the intervention. ${ }^{27,37,48,49,52}$ Two studies demonstrated improvements in dual-task cognitive performance that were retained at 2 weeks ${ }^{48,49}$ and 1 month postintervention. ${ }^{48}$ Three studies showed some degree of retention in dual-task motor performance for periods ranging from 2 months to 6 months postintervention..$^{27,37,52}$

\section{Transfer}

Eight studies assessed whether training benefits transferred to untrained tasks. ${ }^{26,34,36,38,41,45,47,52}$ Three studies ${ }^{26,38,52}$ examined the effect of dual-task postural control training on novel or untrained dual-task postural control tasks. Silsupadol et al ${ }^{26,52}$ showed no transfer effect, while Yamada et $a l^{38}$ demostrated a transfer effect. Two studies ${ }^{41,47}$ examined whether single training benefits transferred to dual-task postural control, and showed negative results. Three studies ${ }^{34,36,45}$ measured the effect of cognitive dual-task training on dual-task performance involving a postural control task and showed transfer to some aspects of dual-task performance (see Table 3).

\section{Discussion}

This investigation of the literature on dual-task training demonstrates the potential to increase postural control, thereby improving balance and walking ability in older adults. Furthermore, this systematic review builds on previous research by examining specific training parameters that may impact the efficacy of dual-task interventions.

\section{Training specificity}

Overall, evidence supports the effectiveness of specific training to improve dual-task postural control among healthy older adults. Training specificity is a key element of motor learning. ${ }^{55}$ However, the definition of training specificity is not obvious within the dual-task paradigm since the intended outcome of interventions could include either an improved ability to divide attention between both tasks or to preferentially improve performance of the postural control task. The majority of studies that incorporated direct dual-task training demonstrated improvement on some aspect of dual-task postural control, with only one exception. ${ }^{43}$ Interventions that trained single-task postural control demonstrated improvement on measures of single-task balance and walking but not on dual-task postural control, with one exception. ${ }^{39}$ Thus, training dual-task performance specifically, rather than just 
single-task performance, appears to be a crucial element for interventions that aim to improve dual-task performance. This notion was supported by several studies with the highest level of evidence included in the review. ${ }^{26,27,33-35,37,38}$

\section{Training content}

The interventions that directly trained dual-task postural control employed a variety of task combinations with different levels of difficulty in the postural control and concurrent cognitive or motor tasks. Some task combinations required mainly mathematical skills for the cognitive task ${ }^{26,27,52}$ whereas others required verbal and memory skills ${ }^{41}$ or auditory skills. ${ }^{37}$ For the postural control task, most studies used walking ${ }^{26,27,40,46}$ or standing ${ }^{35,42,49,50}$ whereas a few used more complicated tasks such as walking within a narrow path ${ }^{26}$ or obstacle crossing. ${ }^{43}$ Two studies used two motor tasks such as standing while catching and throwing a ball, ${ }^{40}$ and walking while holding a tray. ${ }^{39}$ The only study ${ }^{43}$ that specifically trained dual-task performance but did not show specific dual-task performance improvements identified a lack of specificity of outcome measures relative to the trained tasks as well as insufficient training hours (4 hours total) as potential explanations for this finding. While the current research suggests that a variety of trained task combinations can improve dual-task performance, future studies should compare how different combinations of tasks impact the efficacy of training.

The impact of specific task combinations on dual-task performance has been widely discussed. Recent reviews ${ }^{30,31}$ have examined the effect of different concurrent tasks on walking. Al-Yahya et $\mathrm{al}^{31}$ reported that tasks involving internal interference (ie, requiring top-down processing and driven by factors that are internal to the participant ${ }^{56}$ such as verbal fluency or mathematical tasks) have a greater influence on gait parameters than external interference or bottom-up tasks (eg, reaction time tasks). Chu et $\mathrm{al}^{30}$ assessed the predictive value of different task combinations for predicting falls. Their meta-analysis indicated that the combination of a mental tracking task and walking is a good predictor for falls among the elderly. Among the studies in this review, only seven studies used this combination in either the training protocol ${ }^{26,27,38,43,46,52}$ or outcome measure. ${ }^{26,27,36,43,45,47,52}$ Since fall prevention is an important goal of dual-task interventions, future studies should consider incorporating mental tracking tasks in combination with walking in their protocol and/or outcome measurement.

\section{Instructions and feedback}

Variable priority instructions, in which participants were asked to shift their attention back and forth between tasks, appeared to be more effective for improving performance than fixed priority instructions, in which participants focused on either the postural control task or the concurrent cognitive or motor task. ${ }^{26,27,52}$ However, this direct comparison of instructions was limited to only three studies, one of which was a case series with only three participants. Thus, determining the most effective instructions for dual-task training merits further investigation. ${ }^{57}$

Moreover, the effect of feedback ${ }^{55}$ was not explored by any of these investigations, and different forms of feedback may influence motor learning differently among older adults. ${ }^{58}$ Feedback focused on knowledge of results (for example, how many meters someone walks) is more effective than feedback focused on knowledge of performance (the nature of the movement). Tailored feedback during dual-task training could target each task separately or both tasks simultaneously. Nevertheless, even the effect of feedback during motor learning of a single task among the elderly was not clear. ${ }^{59}$ Several issues should be addressed regarding the optimal use of feedback during dual-task training, including: 1) whether feedback is more effective than the absence of feedback during dual-task training among the elderly; and 2) whether feedback on one task at a time is more effective that feedback on both tasks simultaneously during dual-task training.

\section{Training parameters reflecting motor learning}

Several parameters of dual-task training may promote motor learning. The setting of training may influence efficacy. The interventions reviewed here were conducted in two distinct settings: group training and one-on-one training. Similar rates of success were found in both settings; ten out of 13 interventions conducted in a group setting $26,27,33,35,37-40,42,44,51$ demonstrated improvement in some aspects of dual-task, while six out of ten conducted in one-on-one settings ${ }^{34,36,45,46,48,49}$ demonstrated successful outcomes. The dose of intervention is an important factor influencing motor learning. The total training hours varied from 5 hours $^{48}$ to 25 hours $^{37}$ and were spread from 1 week to over 25 weeks. ${ }^{37}$ For studies that showed improvement in dual-task performance, the length of training sessions ranged from 20 minutes $^{38}$ to 60 minutes. ${ }^{33-37,39-42,47,49-51}$ Among studies with the highest methodological quality, Silsupadol et $\mathrm{al}^{26,27}$ conducted the most intensive training, with three sessions per week for 1 month, but Trombetti et a ${ }^{37}$ conducted the longest intervention, with one session per week for 25 weeks. Both of these studies demonstrated some degree of improvement in dual-task walking, with 
some retention of benefits, providing support for a high training dose. Among the studies in this review, the variability in training dose, including session intensity, session frequency, and training duration, makes determining the optimal dose of dual-task training challenging.

\section{Outcome measures}

There is no gold standard for dual-task assessment, and the studies in this review used various dual-task combinations for their outcome measures. For example, the postural control tasks included obstacle crossing, ${ }^{26,43}$ over ground walking, ${ }^{34,40,44,45}$ or standing on a force plate. ${ }^{48}$ Within similar tasks, a variety of different parameters were assessed. For example, the parameters used to assess performance during walking included frontal plane inclination angle, ${ }^{26}$ gait variability, ${ }^{37,39}$ or gait speed. ${ }^{27,40,45}$

Recent research emphasizes the importance of calculating dual-task cost to understand the underlying mechanism of improvement in dual-task performance ${ }^{7}$ and as a sensitive means of assessing fall risk. ${ }^{13,14}$ Dual-task cost is often calculated using the formula:

$$
(\text { dual-task }- \text { single task)/single task } \times 100
$$

and expressed as percentage. Using dual-task cost elucidates the mechanisms underlying the improvement by demonstrating whether improvements are achieved in both tasks or whether improvements in one task occur at the expense of the other task. ${ }^{7}$ Among the studies included in the current review, only three $\mathrm{e}^{41,43,47}$ calculated the dual-task cost; therefore, comparisons between studies are limited. Agmon et $\mathrm{al}^{47}$ showed that there was a change in the trade-off between cognitive and motor costs between pre- and postintervention. This finding demonstrates changes in prioritization between tasks, but not actual improvement with training.

\section{Retention}

Retention of motor skills for up to 1 year has been demonstrated in humans in a laboratory setting. ${ }^{60}$ Among the studies demonstrating the highest level of evidence, Trombetti et $\mathrm{al}^{37}$ demonstrated the longest retention (12 months), although this was only a partial retention of improvements. Future studies should investigate which practice conditions promote optimal retention as well as the effects of interventions that incorporate ongoing or maintenance programs.

\section{Transfer}

Some studies included in this review demonstrated evidence of transfer from trained tasks to novel tasks, but this finding was not uniform. Several studies demonstrated that dual-task postural control training transferred to improvements in novel dual-task combinations. ${ }^{26,38,52}$ Interestingly, three studies that trained cognitive dual-task performance demonstrated improvements on dual-task postural control. ${ }^{34,36,45}$ Participants in these studies were trained, while sitting, on tasks that required switching and dividing attention, and the impact on dual-task walking ${ }^{36,45}$ and standing ${ }^{34}$ was assessed. These studies illuminate the potential to improve dual-task postural control by training two cognitive tasks; a protocol that emphasizes the ability to divide or rapidly shift attention. However, these findings were not consistent and may depend on the level of difficulty of the trained tasks compared to the measured task. ${ }^{43}$

\section{Future directions}

This review highlights several questions that merit further exploration. First, although the existing research provides support for the ability to improve dual-task performance, particularly following response to specific dual-task training, it is not clear what effects these improvements have on function in daily life or fall risk. Outcome measures could be expanded to include functional measurements of dual-task performance relevant to daily life, such as putting on a shirt while standing or walking while talking on the phone. In order to understand the influence of dual-task interventions on falls prevention, future studies should incorporate prospective falls assessments over longer-term follow-up periods.

Second, there is a need to further examine the effect of different motor learning parameters, and the interaction between them, on dual-task acquisition, retention, and transfer. These might include the influence of instructions or different modes of feedback, the specificity of training, and the effect of dose on the response to training. In addition, further exploration is needed to determine the efficacy of training within subgroups of older adults, such as those with and without a history of falls or with different cognitive abilities.

Third, Li et $\mathrm{al}^{61}$ emphasized the importance of adopting an ecological perspective when training and measuring dual-task performance. Thus, finding new ways to address dual-task performance in valid ecological environments needs to be explored. Recently, Mirelman et al ${ }^{62}$ suggested a treadmill with a virtual reality protocol aimed at fall reduction. Such protocols should be tested first on subjects in clinical settings, followed by testing in home-based users. Home-based virtual reality training has the potential to reach larger populations in a complex and safe environment. ${ }^{63}$ 
Finally, in order to strengthen the evidence base for improving dual-task postural control, future studies should include larger, more representative samples and use a standard set of outcome measures to allow cross-study comparison. Outcome measures should include walking speed and stride-to-stride variability, ${ }^{7}$ standard cognitive tasks (such as those that require mental tracking ${ }^{30}$ or internal interference processing), ${ }^{31}$ and calculation of dual-task cost for both tasks. $^{64}$

\section{Limitations}

Sixteen randomized clinical trials ${ }^{26,27,33-46}$ evaluated the effectiveness of training on dual-task performance on a total of 516 subjects with heterogeneous measures that precluded quantitative synthesis or meta-analysis. Furthermore, the quality of evidence in the present review was mixed, with a risk of bias because some studies did not use randomization. The sample was predominately female, limiting the ability to investigate sex differences in intervention efficacy.

Studies incorporated different intervention protocols and various outcome measures to assess the effectiveness of the interventions. This variability made it difficult to identify specific recommendations about the optimal content or duration of dual-task interventions. The lack of long-term follow-up limits the ability to determine whether the benefits of these interventions were retained, as well as the ability to understand the impact of dual-task postural control training on fall risk.

\section{Conclusion}

A synthesis of research examining the effect of different interventions on dual-task postural control suggests that interventions training for balance under single-task conditions can improve balance under single-task conditions, but this improvement does not transfer to dual-task performance. ${ }^{41,47,50}$ Instead, dual-task training appears to be necessary to improve dual-task performance. While variability amongst studies makes it difficult to identify optimal parameters of interventions, it appears that effective interventions can be conducted in either group or one-on-one settings, with a variety of task combinations incorporated into the intervention. The shortest training schedule of 20 minutes twice a week for 24 weeks ${ }^{38}$ as well as only five sessions of 1 hour each ${ }^{34}$ demonstrated improvement in some aspects of dual-task performance.

Future investigations of interventions to improve dualtask postural control should include focused dual-task training and address tasks that have the highest correlation with fall risk. Moreover, long term follow-up with regard to fall occurrence and daily function should be incorporated in order to better understand whether improved dual-task postural control impacts these areas. Future research should also focus on motor learning elements that may extend the retention of dual-task training benefits in order to determine the most effective protocols. Finally, in order to achieve comparability between interventions, an agreed-upon set of outcome measures should be defined and dual-task cost calculation should be included.

\section{Disclosure}

The authors report no conflicts of interest in this work.

\section{References}

1. Rubenstein LZ. Falls in older people: epidemiology, risk factors and strategies for prevention. Age Ageing. 2006;35 Suppl 2:ii37-ii41.

2. Maki BE, Cheng KC, Mansfield A, et al. Preventing falls in older adults: new interventions to promote more effective change-in-support balance reactions. J Electromyogr Kinesiol. 2008;18(2):243-254.

3. Inzitari M, Baldereschi M, Di Carlo A, et al. Impaired attention predicts motor performance decline in older community-dwellers with normal baseline mobility: results from the Italian Longitudinal Study on Aging (ILSA). J Gerontol A Biol Sci Med Sci. 2007;62(8):837-843.

4. Wood KM, Edwards JD, Clay OJ, Wadley VG, Roenker DL, Ball KK. Sensory and cognitive factors influencing functional ability in older adults. Gerontology. 2005;51(2):131-141.

5. Campbell A, Reinken J, Allan B, Martinez G. Falls in old age: a study of frequency and related clinical factors. Age Ageing. 1981;10(4): 264-270.

6. Nevitt MC, Cummings SR, Kidd S, Black D. Risk factors for recurrent nonsyncopal falls. JAMA. 1989;261(18):2663-2668.

7. Montero-Odasso M, Verghese J, Beauchet O, Hausdorff JM. Gait and cognition: a complementary approach to understanding brain function and the risk of falling. J Am Geriatr Soc. 2012;60(11):2127-2136.

8. Fuller GF. Falls in the elderly. Am Fam Physician. 2000;61(7): 2159-2168.

9. Shumway-CookA, Silver IF, LeMier M, York S, Cummings P, KoepsellTD. Effectiveness of a community-based multifactorial intervention on falls and fall risk factors in community-living older adults: a randomized, controlled trial. J Gerontol A Biol Sci Med Sci. 2007;62(12): $1420-1427$.

10. Woollacott M, Shumway-Cook A. Attention and the control of posture and gait: a review of an emerging area of research. Gait Posture. 2002;16(1):1-14.

11. Lundin-Olsson L, Nyberg L, Gustafson Y. "Stops walking when talking" as a predictor of falls in elderly people. Lancet. 1997;349(9052):617.

12. Doumas M, Rapp MA, Krampe RT. Working memory and postural control: adult age differences in potential for improvement, task priority, and dual tasking. J Gerontol B Psychol Sci Soc Sci. 2009;64(2): 193-201.

13. Yamada M, Aoyama T, Arai H, et al. Dua task walk is a reliable predictor of falls in robust elderly adultas. J Am Geriatr Soc. 2011;59(1): 163-164.

14. Beauchet $\mathrm{O}$, Annweiler C, Dubost V, et al. Stops walking when talking: a predictor of falls in older adults? Eur J Neurol. 2009;16(7):786-795.

15. Hausdorff JM, Rios DA, Edelberg HK. Gait variability and fall risk in community-living older adults: a 1-year prospective study. Arch Phys Med Rehabil. 2001;82(8):1050.

16. Lezak M, editor. Neuropsychological Assessment. New York, NY: Oxford University Press; 2004.

17. Royall DR, Palmer R, Chiodo LK, Polk MJ. Declining executive control in normal aging predicts change in functional status: the Freedom House Study. J Am Geriatr Soc. 2004;52(3):346-352. 
18. Springer S, Giladi N, Peretz C, Yogev G, Simon ES, Hausdorff JM. Dual-tasking effects on gait variability: The role of aging, falls, and executive function. Mov Disord. 2006;21(7):950-957.

19. van Iersel MB, Kessels RP, Bloem BR, Verbeek AL, Rikkert MG. Executive functions are associated with gait and balance in communityliving elderly people. J Gerontol A Biol Sci Med Sci. 2008;63(12): 1344-1349.

20. Coppin AK, Shumway-Cook A, Saczynski JS, et al. Association of executive function and performance of dual-task physical tests among older adults: analyses from the InChianti study. Age Ageing. 2006;35(6): 619-624.

21. Zijlstra A, Ufkes T, Skelton D, Lundin-Olsson L, Zijlstra W. Do dual tasks have an added value over single tasks for balance assessment in fall prevention programs? A mini-review. Gerontology. 2008;54(1):40-49.

22. Nordin E, Moe-Nilssen R, Ramnemark A, Lundin-Olsson L. Changes in step-width during dual-task walking predicts falls. Gait Posture. 2010;32(1):92-97.

23. Muhaidat J, Kerr A, Evans JJ, Pilling M, Skelton DA. Validity of simple gait-related dual-task tests in predicting falls in community-dwelling older adults. Arch Phys Med Rehabil. 2014;95(1):58-64.

24. Wollesen B, Voelcker-Rehage C. Training effects on motor-cognitive dual-task performance in older adults. Eur Rev Aging Phys Act 2013:1-20.

25. Beurskens R, Bock O. Age-related deficits of dual-task walking: a review. Neural Plast. 2012;2012:131608.

26. Silsupadol P, Lugade V, Shumway-Cook A, et al. Training-related changes in dual-task walking performance of elderly persons with balance impairment: a double-blind, randomized controlled trial. Gait Posture. 2009;29(4):634-639.

27. Silsupadol P, Shumway-Cook A, Lugade V, et al. Effects of single-task versus dual-task training on balance performance in older adults: a double-blind, randomized controlled trial. Arch Phys Med Rehabil. 2009;90(3):381-387.

28. Shumway-Cook A, Woollacott MH. Aging and postural control. In: Shumway-Cook A, Woollacott MH, editors. Motor Control Translating Research into Clinical Practice. 3rd ed. Philadelphia, PA: Lippincott Williams \& Wilkins; 2007:212-232.

29. Wulf G. Attention and Motor Skill Learning. Champaign, IL: Human Kinetics Publishers; 2007.

30. Chu YH, Tang PF, Peng YC, Chen HY. Meta analysis of type and complexity of a secondary task during walking on the prediction of elderly falls. Geriatr Gerontol Int. 2013;13(2):289-297.

31. Al-Yahya E, Dawes H, Smith L, Dennis A, Howells K, Cockburn J. Cognitive motor interference while walking: a systematic review and meta-analysis. Neurosci Biobehav Rev. 2011;35(3):715-728.

32. Portney LG, Watkins MP. Foundations of Clinical Research: Applications to Practice. 3rd ed: Upper Saddle River, NJ: Pearson and Prentice Hall; 2009.

33. Hiyamizu M, Morioka S, Shomoto K, Shimada T. Effects of dual task balance training on dual task performance ability in elderly people: a randomized controlled trial. Clin Rehabil. 2012;26(1):58-67.

34. Li KZ, Roudaia E, Lussier M, Bherer L, Leroux A, McKinley PA. Benefits of cognitive dual-task training on balance performance in healthy older adults. J Gerontol A Biol Sci Med Sci. 2010;65(12): 1344-1352.

35. Melzer I, Oddsson LIe. Improving balance control and self-reported lower extremity function in community-dwelling older adults: a randomized control trial. Clin Rehabil. 2013;27(3):195-206.

36. Mozolic JL, Long AB, Morgan AR, Rawley-Payne M, Laurienti PJ. A cognitive training intervention improves modality-specific attention in a randomized controlled trial of healthy older adults. Neurobiol Aging. 2011;32(4):655-668

37. Trombetti A, Hars M, Herrmann FR, Kressig RW, Ferrari S, Rizzoli R. Effect of music-based multitask training on gait, balance, and fall risk in elderly people: a randomized controlled trial. Arch Intern Med 2011;171(6):525-533.
38. Yamada M, Aoyama T, Hikita Y, et al. Effects of a DVD-based seated dual-task stepping exercise on the fall risk factors among communitydwelling elderly adults. Telemed $J$ E Health. 2011;17(10):768-772.

39. Donath L, Faude O, Bridenbaugh SA, et al. Transfer effects of fall training on balance performance and spatio-temporal gait parameters in healthy community-dwelling seniors: a pilot study. J Aging Phys Act. Epub July 22, 2013.

40. Granacher U, Muehlbauer T, Bridenbaugh S, Bleiker E, Wehrle A, Kressig RW. Balance training and multi-task performance in seniors. Int J Sports Med. 2010;31(5):353-358.

41. Hall CD, Miszko T, Wolf SL. Effects of Tai Chi intervention on dualtask ability in older adults: a pilot study. Arch Phys Med Rehabil. 2009;90(3):525-529.

42. Pichierri G, Coppe A, Lorenzetti S, Murer K, de Bruin ED. The effect of a cognitive-motor intervention on voluntary step execution under single and dual task conditions in older adults: a randomized controlled pilot study. Clin Interv Aging. 2012;7:175-184.

43. Plummer-D'Amato P, Cohen Z, Daee NA, Lawson SE, Lizotte MR, Padilla A. Effects of once weekly dual-task training in older adults: A pilot randomized controlled trial. Geriatr Gerontol Int. 2012;12(4): 622-629.

44. Uemura K, Yamada M, Nagai K, et al. Effects of dual-task switch exercise on gait and gait initiation performance in older adults: Preliminary results of a randomized controlled trial. Arch Gerontol Geriatr. 2012;54(2):e167-e171.

45. Verghese J, Mahoney J, Ambrose AF, Wang C, Holtzer R. Effect of cognitive remediation on gait in sedentary seniors. $J$ Gerontol A Biol Sci Med Sci. 2010;65(12):1338-1343.

46. You JH, Shetty A, Jones T, Shields K, Belay Y, Brown D. Effects of dual-task cognitive-gait intervention on memory and gait dynamics in older adults with a history of falls: a preliminary investigation. Neuro Rehabilitation. 2009;24(2):193-198.

47. Agmon M, Kelly VE, Logsdon RG, Nguyen H, Belza B. The effect of enhanced fitness (EF) training on dual-task walking in older adults. J Appl Gerontol. Epub December 11, 2012.

48. Bisson E, Contant B, Sveistrup H, Lajoie Y. Functional balance and dual-task reaction times in older adults are improved by virtual reality and biofeedback training. Cyberpsychol Behav. 2007;10(1): $16-23$.

49. Lajoie Y. Effect of computerized feedback postural training on postural and attentional demends in older adults. Aging Clin Exp Res. 2004;16(5):363-368.

50. Melzer I, Marx R, Kurz I. Regular exercise in the elderly is effective to preserve the speed of voluntary stepping under single-task condition but not under dual-task condition. Gerontology. 2009;55(1): $49-57$.

51. Toulotte C, Thevenon A, Fabre C. Effects of training and detraining on the static and dynamic balance in elderly fallers and non-fallers: a pilot study. Disabil Rehabil. 2006;28(2):125-133.

52. Silsupadol P, Siu KC, Shumway-Cook A, Woollacott MH. Training of balance under single- and dual-task conditions in older adults with balance impairment. Phys Ther. 2006;86(2):269-281.

53. Kramer AF, Larish JF, Strayer DL. Training for attentional control in dual task setting: A comparison of young and old adults. J Exp Psychol Appl. 1995;1(1):50-76.

54. Hall E, Hylemon P, Vlahcevic Z, et al. Overexpression of CYP27 in hepatic and extrahepatic cells: role in the regulation of cholesterol homeostasis. Am J Physiol Gastrointest Liver Physiol. 2001;281(1): G293-G301

55. Magill RA. Motor Learning and Control: Concepts and Applications. Volume 11. New York: McGraw-Hill; 2007.

56. Awh E, Belopolsky AV, Theeuwes J. Top-down versus bottom-up attentional control: A failed theoretical dichotomy. Trends Cogn Sci. 2012;16(8):437-443.

57. Del Rosario N. Reasons to Switch: The Effects of Priority and Information Presentation on Dual-Task Interleaving Strategies [master's dissertation]. London: University College London; 2009. 
58. Shea $\mathrm{CH}$, Wulf G. Enhancing motor learning through external-focus instructions and feedback. Hum Mov Sci. 1999;18(4):553-571.

59. Voelcker-Rehage C. Motor-skill learning in older adults - a review of studies on age-related differences. Eur Rev Aging Phys Act. 2008;5(1):5-16.

60. Dayan E, Cohen LG. Neuroplasticity subserving motor skill learning. Neuron. 2011;72(3):443-454.

61. Li KZH, Krampe RT, Bondar A. An ecological approach to studying aging and dual-task performance. Cognitive Limitations in Aging and Psychopathology. 2005:190-218.

62. Mirelman A, Rochester L, Reelick M, et al. V-TIME: a treadmill training program augmented by virtual reality to decrease fall risk in older adults: study design of a randomized controlled trial. BMC Neurol. 2013;13(1):15.
63. Holmes JD, Gu ML, Johnson AM, Jenkins ME. The effects of a home-based virtual reality rehabilitation program on balance among individuals with Parkinson's disease. Phys Occup Ther Geriatr. 2013;31(3):241-253.

64. Kelly VE, Janke AA, Shumway-Cook A. Effects of instructed focus and task difficulty on concurrent walking and cognitive task performance in healthy young adults. Exp Brain Res. 2010;207(1-2):65-73.

\section{Publish your work in this journal}

Clinical Interventions in Aging is an international, peer-reviewed journal focusing on evidence-based reports on the value or lack thereof of treatments intended to prevent or delay the onset of maladaptive correlates of aging in human beings. This journal is indexed on PubMed Central, MedLine, the American Chemical Society's 'Chemical Abstracts
Service' (CAS), Scopus and the Elsevier Bibliographic databases. The manuscript management system is completely online and includes a very quick and fair peer-review system, which is all easy to use. Visit http://www.dovepress.com/testimonials.php to read real quotes from published authors.

Submit your manuscript here: http://www.dovepress.com/clinical-interventions-in-aging-journal 